\title{
CpG-free plasmids confer reduced inflammation and sustained pulmonary gene expression.
}

\begin{abstract}
Pulmonary delivery of plasmid DNA (pDNA)/cationic liposome complexes is associated with an acute unmethylated $\mathrm{CG}$ dinucleotide $(\mathrm{CpG})$-mediated inflammatory response and brief duration of transgene expression. We demonstrate that retention of even a single $\mathrm{CpG}$ in pDNA is sufficient to elicit an inflammatory response, whereas $\mathrm{CpG}$-free pDNA vectors do not. Using a CpG-free pDNA expression vector, we achieved sustained ( $\geq 56 \mathrm{~d}$ ) in vivo transgene expression in the absence of lung inflammation.
\end{abstract}

Keyword: CpG; Gene therapy; Inflammation; Lung; Plasmids. 